\title{
A New Multigrid Finite Element Method for the Transmission Eigenvalue Problems
}

\author{
Jiayu Han, Yidu Yang, Hai Bi \\ School of Mathematics and Computer Science, \\ Guizhou Normal University, Guiyang, 550001, China \\ hanjiayu126@126.com,ydyang@gznu.edu.cn, bihaimath@gznu.edu.cn
}

\begin{abstract}
Numerical methods for the transmission eigenvalue problems are hot topics in recent years. Based on the work of Lin and Xie [Math. Comp., 84(2015), pp. 71-88], we build a multigrid method to solve the problems. With our method, we only need to solve a series of primal and dual eigenvalue problems on a coarse mesh and the associated boundary value problems on the finer and finer meshes. Theoretical analysis and numerical results show that our method is simple and easy to implement and is efficient for computing real and complex transmission eigenvalues.
\end{abstract}

Keywords : Transmission eigenvalues, Multigrid method, Nonsymmetric eigenvalue problems, Extended/Generalized finite element.

\section{Introduction}

The transmission eigenvalue problems have theoretical importance in the uniqueness and reconstruction in inverse scattering theory [1, 2]. Transmission eigenvalues can be determined from the far-field data of the scattered wave and used to obtain estimates for the material properties of the scattering object [3, 4. Many literatures such as 2, 4, 5, 6, 7, studied the existence of transmission eigenvalues, and [4, 8, 9] et al. explored the upper and lower bounds for the index of refraction $n(x)$.

In recent years, numerical methods for the transmission eigenvalue problems have attracted the attention from more and more researchers. The first numerical treatment of the transmission eigenvalue problem appeared in [10] where three finite element methods are proposed for the Helmholtz transmission eigenvalues. Later on, many other numerical methods were developed to solve the problems (see, e.g., 11, 12, 13, 14, 15]). In particular, Sun [1] proposed an iterative method and gave a coarse error analysis. Furthermore, Ji et al. [12] developed his work and proved the accurate error estimates for both eigenvalues and eigenfunctions by constructing an auxiliary problem as a bridge. An and 
Shen [13] proposed a spectral-element method to solve this problem numerically. Afterwards using the linearized technique the authors in [14, 15] builded two new weak formulations and the corresponding finite element discretizations.

The idea of multigrid methods for eigenvalue problems was developed originally from two grid methodology. In 2001, Xu and Zhou [16 proposed a two grid method based on inverse iteration for elliptic eigenvalue problems, which is, in a way, related to that in Lin and Xie [17. After that, the two grid method was further developed into multigrid method and local and parallel algorithm in [18, 19, 20, 21] et al. In recent years, Lin and Xie 22, 23. proposed a mutilevel correction method. This method can be regarded as the combination of two grid method and the extended/generalized finite element method. The extended/generalized finite element method was developed in 1990s by 24, 25, 26, 27] et al, which has important applications on problems in material science [28, 29]. The method of Lin and Xie [22, 23] enriches the finite element space at each correction step with the numerical eigenfunctions obtained from the last step. So it is able to naturally reproduce the feature of eigenfunctions: the discontinuity, singularity, boundary layer, etc. Such an embedding of the problem's feature into the finite element space can significantly improve convergence rates and accuracy step by step, so that the multigrid method can achieve the same accuracy as solving the eigenvalue problem directly but with less computational work.

In this paper, based on the literatures [22, 23, we propose a new multigrid method to solve the transmission eigenvalue problems but based on the new weak formulation (2.6) proposed in [15] which is a linear and nonsymmetric eigenvalue problem. In this work, (1) we prove the error estimates of the transmission eigenvalues and eigenfunctions for our multigrid method. Our theoretical results are valid for arbitrary real and complex eigenvalues. (2) With our method, due to adopting the linearized weak formulation, we can transform the transmission eigenvalue problem into a generalized matrix eigenvalue problem and can be solved efficiently by the sparse solver eigs in Matlab; (3) with our multigrid method, the solution of eigenvalue problem on a fine mesh can be reduced to a series of the solutions of the eigenvalue problem on a coarse meshes and a series of solutions of the boundary value problems on the multilevel meshes. As numerical results indicate, this method is applicable to the real and complex transmission eigenvalues.

\section{Preliminaries}

Let $H^{s}(D)$ be a Sobolev space with norm $\|\cdot\|_{s}(s=1,2)$, and

$$
H_{0}^{2}(D)=\left\{v \in H^{2}(D):\left.v\right|_{\partial D}=\left.\frac{\partial v}{\partial \nu}\right|_{\partial D}=0\right\} .
$$

Consider the Helmholtz transmission eigenvalue problem: Find $k \in \mathcal{C}, \omega, \sigma \in$ $L^{2}(D), \omega-\sigma \in H^{2}(D)$ such that

$$
\begin{aligned}
& \Delta \omega+k^{2} n(x) \omega=0, \quad \text { in } D, \\
& \Delta \sigma+k^{2} \sigma=0, \quad \text { in } D, \\
& \omega-\sigma=0, \quad \text { on } \partial D, \\
& \frac{\partial \omega}{\partial \nu}-\frac{\partial \sigma}{\partial \nu}=0, \quad \text { on } \partial D,
\end{aligned}
$$


where $D \subset \mathbb{R}^{2}$ or $D \subset \mathbb{R}^{3}$ is a bounded simply connected inhomogeneous medium, $\nu$ is the unit outward normal to $\partial D$.

It is possible to write (2.1)-(2.4) as an equivalent eigenvalue problem for $u=\omega-\sigma \in H_{0}^{2}(D)$. In particular,

$$
\left(\Delta u+k^{2} u\right)=\Delta \omega+k^{2} \omega=k^{2}(1-n) \omega .
$$

Dividing by $n-1$ and applying the operator $\left(\Delta+k^{2} n\right)$ to the above equality, the eigenvalue problem (2.1)-(2.4) can be stated as follows: Find $k^{2} \in \mathcal{C}, k^{2} \neq 0$, nontrivial $u \in H_{0}^{2}(D)$ such that

$$
\left(\frac{1}{n(x)-1}\left(\Delta u+k^{2} u\right), \Delta v+k^{2} n(x) v\right)_{0}=0, \quad \forall v \in H_{0}^{2}(D),
$$

where $(\cdot, \cdot)_{0}$ is the inner product of $L^{2}(D)$. As usual, we define $\lambda=k^{2}$ as the transmission eigenvalue in this paper. We suppose that the index of refraction $n \in L^{\infty}(D)$ satisfying either one of the following assumptions

$$
\begin{aligned}
& (C 1) \quad 1+\delta \leq \inf _{D} n(x) \leq n(x) \leq \sup _{D} n(x)<\infty, \\
& (C 2) \quad 0<\inf _{D} n(x) \leq n(x) \leq \sup _{D} n(x)<1-\varrho,
\end{aligned}
$$

for some constant $\delta>0$ or $\varrho>0$.

For simplicity, in the coming discussion we assume $(C 1)$ holds and $n(x)$ is proper smooth (for example $n(x) \in W^{2, \infty}(D)$ ). For the case $(C 2)$ the argument method is the same.

Define Hilbert space $\mathbf{H}=H_{0}^{2}(D) \times L^{2}(D)$ with norm $\|(u, w)\|_{\mathbf{H}}=\|u\|_{2}+$ $\|w\|_{0}$, and define $\mathbf{H}_{s}=H^{s}(D) \times H^{s-2}(D)$ with norm $\|(u, w)\|_{\mathbf{H}_{s}}=\|u\|_{s}+$ $\|w\|_{s-2}, s=0,1$.

From (2.5) we derive that

$$
\begin{aligned}
& \left(\frac{1}{n-1} \Delta u, \Delta v\right)_{0}-\lambda\left(\nabla\left(\frac{1}{n-1} u\right), \nabla v\right)_{0} \\
& \quad-\lambda\left(\nabla u, \nabla\left(\frac{n}{n-1} v\right)\right)_{0}+\lambda^{2}\left(\frac{n}{n-1} u, v\right)_{0}=0, \quad \forall v \in H_{0}^{2}(D) .
\end{aligned}
$$

Let $w=\lambda u$, we arrive at a linear weak formulation: Find $(\lambda, u, w) \in \mathcal{C} \times$ $H_{0}^{2}(D) \times L^{2}(D)$ such that

$$
\begin{aligned}
& \left(\frac{1}{n-1} \Delta u, \Delta v\right)_{0}=\lambda\left(\nabla\left(\frac{1}{n-1} u\right), \nabla v\right)_{0} \\
& \quad+\lambda\left(\nabla u, \nabla\left(\frac{n}{n-1} v\right)\right)_{0}-\lambda\left(\frac{n}{n-1} w, v\right)_{0}, \quad \forall v \in H_{0}^{2}(D), \\
& (w, z)_{0}=\lambda(u, z)_{0}, \quad \forall z \in L^{2}(D) .
\end{aligned}
$$

We introduce the following sesquilinear forms

$$
\begin{aligned}
& A((u, w),(v, z))=\left(\frac{1}{n-1} \Delta u, \Delta v\right)_{0}+(w, z)_{0} \\
& B((u, w),(v, z)) \\
& \quad=\left(\nabla\left(\frac{1}{n-1} u\right), \nabla v\right)_{0}+\left(\nabla u, \nabla\left(\frac{n}{n-1} v\right)\right)_{0}-\left(\frac{n}{n-1} w, v\right)_{0}+(u, z)_{0} \\
& \quad=-\left(\frac{1}{n-1} u, \nabla \cdot \nabla v\right)_{0}-\left(u, \nabla \cdot \nabla\left(\frac{n}{n-1} v\right)\right)_{0}-\left(\frac{n}{n-1} w, v\right)_{0}+(u, z)_{0},
\end{aligned}
$$


then (2.5) can be rewritten as: Find $\lambda \in \mathcal{C}$, nontrivial $(u, w) \in \mathbf{H}$ such that

$$
A((u, w),(v, z))=\lambda B((u, w),(v, z)), \quad \forall(v, z) \in \mathbf{H} .
$$

Let norm $\|\cdot\|_{A}$ be induced by the inner product $A(\cdot, \cdot)$, then it is clear $\|\cdot\|_{A}$ is equivalent to $\|\cdot\|_{\mathbf{H}}$.

One can easily verify that for any given $(f, g) \in \mathbf{H}_{s}(s=0,1), B((f, g),(v, z))$ is a continuous linear form on $\mathbf{H}$ :

$$
B((f, g),(v, z)) \lesssim\|(f, g)\|_{\mathbf{H}_{s}}\|(v, z)\|_{\mathbf{H}}, \forall(v, z) \in \mathbf{H}
$$

Here and hereafter this paper, we use the symbols $x \lesssim y$ to mean $x \leq C y$ for a constant $C$ that is independent of mesh size and iteration times and may be different at different occurrences.

The source problem associated with (2.6) is given by: Find $(\psi, \varphi) \in \mathbf{H}$ such that

$$
A((\psi, \varphi),(v, z))=B((f, g),(v, z)), \quad \forall(v, z) \in \mathbf{H}
$$

From the Lax-Milgram theorem we know that the problem (2.7) exists an unique solution, therefore, we define the corresponding solution operator $T: \mathbf{H}_{s} \rightarrow \mathbf{H}$ by

$$
A(T(f, g),(v, z))=B((f, g),(v, z)), \quad \forall(v, z) \in \mathbf{H} .
$$

Then (2.6) has the equivalent operator form:

$$
T(u, w)=\lambda^{-1}(u, w) .
$$

Consider the dual problem of (2.6): Find $\lambda^{*} \in \mathcal{C}$, nontrivial $\left(u^{*}, w^{*}\right) \in \mathbf{H}$ such that

$$
A\left((v, z),\left(u^{*}, w^{*}\right)\right)=\overline{\lambda^{*}} B\left((v, z),\left(u^{*}, w^{*}\right)\right), \quad \forall(v, z) \in \mathbf{H} .
$$

Note that the primal and dual eigenvalues are connected via $\lambda=\overline{\lambda^{*}}$.

Define the corresponding solution operator $T^{*}: \mathbf{H}_{s} \rightarrow \mathbf{H}$ by

$$
A\left((v, z), T^{*}(f, g)\right)=B((v, z),(f, g)), \quad \forall(v, z) \in \mathbf{H} .
$$

Then (2.10) has the equivalent operator form:

$$
T^{*}\left(u^{*}, w^{*}\right)=\lambda^{*-1}\left(u^{*}, w^{*}\right) .
$$

Clearly, $T^{*}$ is the adjoint operator of $T$ in the sense of inner product $A(\cdot, \cdot)$. In order to discretize the space $\mathbf{H}$, we need two finite element spaces to discretize $H_{0}^{2}(D)$ and $L^{2}(D)$, respectively, but here we can construct only one conforming finite element space $S^{h} \subset H_{0}^{2}(D)$ such that $\mathbf{H}_{h}:=S^{h} \times S^{h} \subset H_{0}^{2}(D) \times L^{2}(D)$.

The conforming finite element approximation of (2.6) is given by the following: Find $\lambda_{h} \in \mathcal{C}$, nontrivial $\left(u_{h}, w_{h}\right) \in \mathbf{H}_{h}$ such that

$$
A\left(\left(u_{h}, w_{h}\right),(v, z)\right)=\lambda_{h} B\left(\left(u_{h}, w_{h}\right),(v, z)\right), \quad \forall(v, z) \in \mathbf{H}_{h} .
$$


We introduce the corresponding solution operator: $T_{h}: \mathbf{H}_{s} \rightarrow \mathbf{H}_{h}(\mathrm{~s}=0,1)$ :

$$
A\left(T_{h}(f, g),(v, z)\right)=B((f, g),(v, z)), \quad \forall(v, z) \in \mathbf{H}_{h} .
$$

Then (2.13) has the operator form:

$$
T_{h}\left(u_{h}, w_{h}\right)=\lambda_{h}^{-1}\left(u_{h}, w_{h}\right) .
$$

Define the projection operators $P_{h}^{1}: H_{0}^{2}(D) \rightarrow S^{h}$ and $P_{h}^{2}: L^{2}(D) \rightarrow S^{h}$ by

$$
\begin{aligned}
& \left(\frac{1}{n-1} \Delta\left(u-P_{h}^{1} u\right), \Delta v\right)_{0}=0, \quad \forall v \in S^{h}, \\
& \left(w-P_{h}^{2} w, z\right)_{0}=0, \quad \forall z \in S^{h} .
\end{aligned}
$$

Let

$$
P_{h}(u, w)=\left(P_{h}^{1} u, P_{h}^{2} w\right), \quad \forall(u, w) \in \mathbf{H} .
$$

Then $P_{h}: \mathbf{H} \rightarrow \mathbf{H}_{h}$, and

$$
A\left((u, w)-P_{h}(u, w),(v, z)\right)=0, \quad \forall(v, z) \in \mathbf{H}_{h} .
$$

We need the following regularity assumption:

$\mathbf{R}(\mathbf{D})$. For any $\xi \in H^{-s}(D)(s=0,1)$, there exists $\psi \in H^{2+r_{s}}(D)$ satisfying

$$
\begin{aligned}
& \Delta\left(\frac{1}{n-1} \Delta \psi\right)=\xi, \quad \text { in } D, \\
& \psi=\frac{\partial \psi}{\partial \nu}=0 \quad \text { on } \partial D,
\end{aligned}
$$

and

$$
\|\psi\|_{2+r_{s}} \leq C_{p}\|\xi\|_{-s}, \quad s=0,1
$$

where $r_{1} \in(0,1], r_{0} \in(0,2], C_{p}$ denotes the prior constant dependent on the equation and $D$ but independent of the right-hand side $\xi$ of the equation.

It is easy to know that (2.19) is valid with $r_{s}=2-s$ when $n \in W^{2, p}(D)$ ( $p$ is greater than but arbitrarily close to 2 ) and $\partial D$ is appropriately smooth. When $D \subset R^{2}$ is a convex polygon, from Theorem 2 in [30], when $n \in W^{2, p}(D)$, we can get $r_{1}=1$ and that if the inner angle at each critical boundary point is smaller than $126.283696 \ldots 0$ then $r_{0}=2$.

The following Lemmas 2.1-2.3 come from [15]. They give in the sense of lower norms the estimates of the finite element projection and the convergence of $T_{h}$ to $T$.

Lemma 2.1 [15, Lemma 3.4]. Suppose that $n \in W^{2, \infty}(D)$ and $\mathbf{R}(\mathbf{D})$ is valid $(s=0,1)$, then for $(u, w) \in \mathbf{H}$,

$$
\left\|(u, w)-P_{h}(u, w)\right\|_{\mathbf{H}_{s}} \lesssim h^{r_{s}}\left\|(u, w)-P_{h}(u, w)\right\|_{\mathbf{H}}, \quad s=0,1 .
$$

The conforming finite element approximation of (2.10) is given by: Find $\lambda_{h}^{*} \in \mathcal{C},\left(u_{h}^{*}, w_{h}^{*}\right) \in \mathbf{H}_{h}$ such that

$$
A\left((v, z),\left(u_{h}^{*}, w_{h}^{*}\right)\right)=\overline{\lambda_{h}^{*}} B\left((v, z),\left(u_{h}^{*}, w_{h}^{*}\right)\right), \quad \forall(v, z) \in \mathbf{H}_{h} .
$$

Note that the primal and dual eigenvalues are connected via $\lambda_{h}=\overline{\lambda_{h}^{*}}$. 
Define the solution operator $T_{h}^{*}: \mathbf{H}_{s} \rightarrow \mathbf{H}_{h}$ satisfying

$$
A\left((v, z), T_{h}^{*}(f, g)\right)=B((v, z),(f, g)), \quad \forall(v, z) \in \mathbf{H}_{h} .
$$

Naturally (2.21) has the following equivalent operator form

$$
T_{h}^{*}\left(u_{h}^{*}, w_{h}^{*}\right)=\lambda_{h}^{*-1}\left(u_{h}^{*}, w_{h}^{*}\right) .
$$

Lemma 2.2 [15, Theorem 3.1]. Let $n \in W^{1, \infty}(D)$, then

$$
\begin{gathered}
\left\|T-T_{h}\right\|_{\mathbf{H}} \rightarrow 0, \\
\left\|T-T_{h}\right\|_{\mathbf{H}_{1}} \rightarrow 0,
\end{gathered}
$$

and let $n \in W^{2, \infty}(D)$, then

$$
\left\|T-T_{h}\right\|_{\mathbf{H}_{0}} \rightarrow 0 .
$$

In this paper, let $\lambda_{i}$ be the ith eigenvalue of (2.6) with the algebraic multiplicity $q$ and the ascent 1 . Then, according to spectral approximation theory [31, 32, there are $q$ eigenvalues $\lambda_{j, h}(j=i, \cdots, i+q-1)$ of (2.13) converging to $\lambda_{i}$. Let $M\left(\lambda_{i}\right)$ be the space spanned by all eigenfunctions corresponding to the eigenvalue $\lambda_{i}$. Let $M_{h}\left(\lambda_{i}\right)$ be the space spanned by all generalized eigenfunctions corresponding to the numerical eigenvalues $\left\{\lambda_{j, h}\right\}_{j=i}^{i+q-1}$ of (2.13). As for the dual problems (2.10) and (2.21), the definitions of $M^{*}\left(\lambda_{i}^{*}\right)$ and $M_{h}^{*}\left(\lambda_{i}^{*}\right)$ are made similarly to $M\left(\lambda_{i}\right)$ and $M_{h}\left(\lambda_{i}\right)$, respectively.

In what follows, to describe the approximation relation between the finite element space $\mathbf{H}_{h}$ and the eigenfunction spaces $M\left(\lambda_{i}\right)$ and $M^{*}\left(\lambda_{i}^{*}\right)$, we introduce the following quantities

$$
\begin{aligned}
& \delta_{h}\left(\lambda_{i}\right)=\sup _{\substack{(v, z) \in M\left(\lambda_{i}\right) \\
\|(v, z)\|_{\mathbf{H}}=1}} \inf _{\left(v_{h}, z_{h}\right) \in \mathbf{H}_{h}}\left\|(v, z)-\left(v_{h}, z_{h}\right)\right\|_{\mathbf{H}}, \\
& \delta_{h}^{*}\left(\lambda_{i}^{*}\right)=\sup _{\substack{(v, z) \in M^{*}\left(\lambda_{i}^{*}\right) \\
\|(v, z)\|_{\mathbf{H}}=1}} \inf _{\left(v_{h}, z_{h}\right) \in \mathbf{H}_{h}}\left\|(v, z)-\left(v_{h}, z_{h}\right)\right\|_{\mathbf{H}} .
\end{aligned}
$$

The operator convergence results (2.24)-(2.26) are critical as a bridge of making the error analysis for the discrete problem (2.13). From these results, we yield immediately the following lemma using the spectral approximation theory.

Lemma 2.3. Suppose $n \in W^{2, \infty}(D)$. Let $\left(u_{j, h}, w_{j, h}\right)(j=i, i+1, \cdots, i+$ $q-1)$ be eigenfunction corresponding to $\lambda_{j, h}$ and $\left\|\left(u_{j, h}, w_{j, h}\right)\right\|_{A}=1$, then there exists eigenfunction $\left(u_{i}, w_{i}\right)$ corresponding to $\lambda_{i}$ such that

$$
\begin{aligned}
& \left\|\left(u_{j, h}, w_{j, h}\right)-(u, w)\right\|_{\mathbf{H}} \lesssim \delta_{h}\left(\lambda_{i}\right), \\
& \left\|\left(u_{j, h}, w_{j, h}\right)-(u, w)\right\|_{\mathbf{H}_{s}} \lesssim h^{r_{s}} \delta_{h}\left(\lambda_{i}\right), s=0,1, \\
& \left|\lambda_{i}-\lambda_{j, h}\right| \lesssim \delta_{h}\left(\lambda_{i}\right) \delta_{h}^{*}\left(\lambda_{i}^{*}\right) .
\end{aligned}
$$

Remark 2.1. The similar estimates as above are valid for the dual problem (2.13) (see [15).

\section{A New Multigrid Method}

In this section, based on the multilevel correction method proposed by Lin and Xie [22, 23, we give the multigrid scheme for the weak form (2.6). Our 
theoretical results are given in Theorems 3.1-3.2. Prior to our argument, we give the following basic condition related to finite element spaces and their approximation relation to eigenfunction spaces.

We construct the finite element spaces such that $\mathbf{H}_{H}=\mathbf{H}_{h_{1}} \subset \mathbf{H}_{h_{2}} \subset \cdots \subset$ $\mathbf{H}_{h_{n}}$ and

$$
\delta_{h_{m+1}} \approx \frac{1}{\beta} \delta_{h_{m}}, \delta_{h_{m+1}}^{*} \approx \frac{1}{\beta} \delta_{h_{m}}^{*},
$$

where $\beta>1$ is a constant only dependent on the smoothness of eigenfunctions corresponding to $\lambda_{i}$ and the degree $t$ of the piecewise polynomial space.

The above condition is readily satisfied on regular meshes. In particular, if the meshes are obtained from a procedure of bisection mesh refinement and $M\left(\lambda_{i}\right), M^{*}\left(\lambda_{i}^{*}\right) \subset H^{2+r}(D) \times H^{r}(D)(r \leq t-1)$, then we have approximately $\beta \approx 2^{r}$.

Assume that we have obtained the eigenpair approximations $\left(\lambda_{j, h_{m}}, u_{j, h_{m}}, w_{j, h_{m}}\right)$ $(j=i, i+1, \cdots, i+q-1)$ and the corresponding dual ones $\left(\lambda_{j, h_{m}}^{*}, u_{j, h_{m}}^{*}, w_{j, h_{m}}^{*}\right)$. First of all, we give one correction step of the multigrid scheme.

Algorithm 1. One Correction Step.

Step 1. Solve the following linear boundary value problems: For $j=i, i+$ $1, \cdots, i+q-1$, find $\left(\widehat{u}_{j, h_{m+1}}, \widehat{w}_{j, h_{m+1}}\right) \in \mathbf{H}_{h_{m+1}}$ such that

$A\left(\left(\widehat{u}_{j, h_{m+1}}, \widehat{w}_{j, h_{m+1}}\right),(v, z)\right)=\lambda_{j, h_{m}} B\left(\left(u_{j, h_{m}}, w_{j, h_{m}}\right),(v, z)\right), \quad \forall(v, z) \in \mathbf{H}_{h_{m+1}}$, and find $\left(\widehat{u}_{j, h_{m+1}}^{*}, \widehat{w}_{j, h_{m+1}}^{*}\right) \in \mathbf{H}_{h_{m+1}}$ such that $A\left((v, z),\left(\widehat{u}_{j, h_{m+1}}^{*}, \widehat{w}_{j, h_{m+1}}^{*}\right)\right)=\lambda_{j, h_{m}} B\left((v, z),\left(u_{j, h_{m}}^{*}, w_{j, h_{m}}^{*}\right)\right), \quad \forall(v, z) \in \mathbf{H}_{h_{m+1}}$.

Step 2. Construct a new finite element space $\mathbf{H}_{H, h_{m+1}} \supseteq \mathbf{H}_{H}+\operatorname{span}\left\{\left(\widehat{u}_{j, h_{m+1}}, \widehat{w}_{j, h_{m+1}}\right)\right.$, $\left.\left(\widehat{u}_{j, h_{m+1}}^{*}, \widehat{w}_{j, h_{m+1}}^{*}\right), j=i, i+1, \cdots, i+q-1\right\}$ and solve the following eigenvalue problems: For $j=i, i+1, \cdots, i+q-1$, find $\lambda_{j, h_{m+1}} \in \mathcal{C}$, nontrivial $\left(u_{j, h_{m+1}}, w_{j, h_{m+1}}\right) \in \mathbf{H}_{H, h_{m+1}}$ such that

$A\left(\left(u_{j, h_{m+1}}, w_{j, h_{m+1}}\right),(v, z)\right)=\lambda_{j, h_{m+1}} B\left(\left(u_{j, h_{m}}, w_{j, h_{m+1}}\right),(v, z)\right), \quad \forall(v, z) \in \mathbf{H}_{H, h_{m+1}}$, and find nontrivial $\left(u_{j, h_{m+1}}^{*}, w_{j, h_{m+1}}^{*}\right) \in \mathbf{H}_{H, h_{m+1}}$ such that $A\left((v, z),\left(u_{j, h_{m+1}}^{*}, w_{j, h_{m+1}}^{*}\right)\right)=\lambda_{j, h_{m+1}} B\left((v, z),\left(u_{j, h_{m+1}}^{*}, w_{j, h_{m+1}}^{*}\right)\right), \quad \forall(v, z) \in \mathbf{H}_{H, h_{m+1}}$.

We output $\left\{\lambda_{j, h_{m+1}}\right\}_{j=i}^{i+q-1}$ and a basis $\left\{\left(u_{j, h_{m+1}}, w_{j, h_{m+1}}\right)\right\}_{j=i}^{i+q-1}$ of $M_{h_{m+1}}\left(\lambda_{i}\right)$ with $\left\|\left(u_{j, h_{m+1}}, w_{j, h_{m+1}}\right)\right\|_{A}=1$ and a basis $\left\{\left(u_{j, h_{m+1}}^{*}, w_{j, h_{m+1}}^{*}\right)\right\}_{j=i}^{i+q-1}$ of $M_{h_{m+1}}^{*}\left(\lambda_{i}^{*}\right)$ with $\left\|\left(u_{j, h_{m+1}}^{*}, w_{j, h_{m+1}}^{*}\right)\right\|_{A}=1$. We define the above two steps as Procedure Correction:

$$
\begin{aligned}
& \left\{\lambda_{j, h_{m+1}}, u_{j, h_{m+1}}, w_{j, h_{m+1}}, u_{j, h_{m+1}}^{*}, w_{j, h_{m+1}}^{*}\right\}_{j=i}^{i+q-1} \\
& =\operatorname{Correction}\left(\mathbf{H}_{H},\left\{\lambda_{j, h_{m}}, u_{j, h_{m}}, w_{j, h_{m}}, u_{j, h_{m}}^{*}, w_{j, h_{m}}^{*}\right\}_{j=i}^{i+q-1}, \mathbf{H}_{h_{m+1}}\right)
\end{aligned}
$$

Implementing Procedure Correction repeatedly leads to the following Scheme. 
Algorithm 2. Mutigrid Scheme.

Step 1. Construct a series of nested finite element spaces $\mathbf{H}_{H}=\mathbf{H}_{h_{1}}, \mathbf{H}_{h_{2}}$, $\cdots, \mathbf{H}_{h_{n}}$ such that (3.1) holds.

Step 2. For $j=i, i+1, \cdots, i+q-1$, find $\left(\lambda_{j, h_{1}}, u_{j, h_{1}}, w_{j, h_{1}}\right) \in \mathcal{C} \times \mathbf{H}_{h_{1}}$ such that $\left\|\left(u_{j, h_{1}}, w_{j, h_{1}}\right)\right\|_{A}=1$ and

$$
A\left(\left(u_{j, h_{1}}, w_{j, h_{1}}\right),(v, z)\right)=\lambda_{j, h_{1}} B\left(\left(u_{j, h_{1}}, w_{j, h_{1}}\right),(v, z)\right), \quad \forall(v, z) \in \mathbf{H}_{h_{1}},
$$

and find $\left(u_{j, h_{1}}^{*}, w_{j, h_{1}}^{*}\right) \in \mathbf{H}_{h_{1}}$ such that $\left\|\left(u_{j, h_{1}}^{*}, w_{j, h_{1}}^{*}\right)\right\|_{A}=1$ and

$$
A\left((v, z),\left(u_{j, h_{1}}^{*}, w_{j, h_{1}}^{*}\right)\right)=\lambda_{j, h_{1}} B\left((v, z),\left(u_{j, h_{1}}^{*}, w_{j, h_{1}}^{*}\right)\right), \quad \forall(v, z) \in \mathbf{H}_{h_{1}} .
$$

Step 3. For $m=1,2, \cdots, N-1$

Obtain a new eigenpair approximation $\left(\lambda_{j, h_{m+1}}, u_{j, h_{m+1}}, w_{j, h_{m+1}}\right)$ by

$$
\begin{aligned}
& \left\{\lambda_{j, h_{m+1}}, u_{j, h_{m+1}}, w_{j, h_{m+1}}, u_{j, h_{m+1}}^{*}, w_{j, h_{m+1}}^{*}\right\}_{j=i}^{i+q-1} \\
& =\text { Correction }\left(\mathbf{H}_{H},\left\{\lambda_{j, h_{m}}, u_{j, h_{m}}, w_{j, h_{m}}, u_{j, h_{m}}^{*}, w_{j, h_{m}}^{*}\right\}_{j=i}^{i+q-1}, \mathbf{H}_{h_{m+1}}\right)
\end{aligned}
$$

End

Before making the error analysis of multigrid scheme, we give the following assumption.

(A) Quasi-biorthogonality. Suppose that there are $\left\{\left(\widetilde{u}_{j, h_{m}}, \widetilde{w}_{j, h_{m}}\right)\right\}_{j=i}^{i+q-1}$ $\subset M_{h_{m}}\left(\lambda_{i}\right)$ with $\left\|\left(\widetilde{u}_{j, h_{m}}, \widetilde{w}_{j, h_{m}}\right)\right\|_{\mathbf{H}}=1$ and $\left\{\left(\widetilde{u}_{j, h_{m}}^{*}, \widetilde{w}_{j, h_{m}}^{*}\right)\right\}_{j=i}^{i+q-1} \in M_{h_{m}}^{*}\left(\lambda_{i}^{*}\right)$ with $\left\|\left(\widetilde{u}_{j, h_{m}}^{*}, \widetilde{w}_{j, h_{m}}^{*}\right)\right\|_{\mathbf{H}}=1$ such that

$$
\left|A\left(\left(u_{j, h_{m}}, w_{j, h_{m}}\right),\left(\widetilde{u}_{l, h_{m}}^{*}, \widetilde{w}_{l, h_{m}}^{*}\right)\right)\right|+\left|A\left(\left(\widetilde{u}_{j, h_{m}}, \widetilde{w}_{j, h_{m}}\right),\left(u_{l, h_{m}}^{*}, w_{l, h_{m}}^{*}\right)\right)\right| \lesssim H^{r_{0}}
$$

$(j, l=i, i+1, \cdots, i+q-1, j \neq l)$,

and $\left|A\left(\left(u_{j, h_{m}}, w_{j, h_{m}}\right),\left(\widetilde{u}_{j, h_{m}}^{*}, \widetilde{w}_{j, h_{m}}^{*}\right)\right)\right|+\left|A\left(\left(\widetilde{u}_{j, h_{m}}, \widetilde{w}_{j, h_{m}}\right),\left(u_{j, h_{m}}^{*}, w_{j, h_{m}}^{*}\right)\right)\right|(j=$ $i, i+1, \cdots, i+q-1)$ has a positive lower bound uniformly with respective to $h_{m}$.

When $\lambda_{i}$ is a simple eigenvalue $(q=1)$, it is clear that Assumption (A) is valid; when $q>1$, we can prove the follow conclusion:

If the distance (in $\left.\|\cdot\|_{\mathbf{H}}\right)$ from $\left(u_{j, h_{m}}, w_{j, h_{m}}\right)(j=i, i+1, \cdots, i+q-1)$ to $\operatorname{span}\left\{\left(u_{l, h_{m}}, w_{l, h_{m}}\right), l=i, i+1, \cdots, i+q-1, l \neq j\right\}$ has a positive lower bound uniformly with respective to $h_{m}$, then Assumption (A) is valid.

The following theorem, an extension of the corresponding theorems in [22, 23, 33, indicates that the accuracy of numerical eigenpair can be apparently improved after one correction step.

Theorem 3.1. Suppose that $(\mathbf{A})$ is valid and the ascent of $\lambda_{j, h_{m}}(j=$ $i, \cdots, i+q-1)$ is 1 , and there exist two eigenpairs $\left(\lambda_{i}, u_{i}, w_{i}\right)$ and $\left(\lambda_{i}^{*}, u_{i}^{*}, w_{i}^{*}\right)$ such that the eigenpair approximations $\left(\lambda_{j, h_{m}}, u_{j, h_{m}}, w_{j, h_{m}}\right),\left(\lambda_{j, h_{m}}^{*}, u_{j, h_{m}}^{*}\right.$, 
$\left.w_{j, h_{m}}^{*}\right) \in \mathcal{C} \times \mathbf{H}_{h_{m}}$ have the following estimates

$$
\begin{aligned}
\left\|\left(u_{j, h_{m}}, w_{j, h_{m}}\right)-\left(u_{j}, w_{j}\right)\right\|_{\mathbf{H}} & \lesssim \varepsilon_{h_{m}}\left(\lambda_{i}\right), \\
\left\|\left(u_{j, h_{m}}, w_{j, h_{m}}\right)-\left(u_{j}, w_{j}\right)\right\|_{\mathbf{H}_{s}} & \lesssim H^{r_{s}} \varepsilon_{h_{m}}\left(\lambda_{i}\right), s=0,1, \\
\left\|\left(u_{j, h_{m}}^{*}, w_{j, h_{m}}^{*}\right)-\left(u_{j}^{*}, w_{j}^{*}\right)\right\|_{\mathbf{H}} & \lesssim \varepsilon_{h_{m}}^{*}\left(\lambda_{i}\right), \\
\left\|\left(u_{j, h_{m}}^{*}, w_{j, h_{m}}^{*}\right)-\left(u_{j}^{*}, w_{j}^{*}\right)\right\|_{\mathbf{H}_{s}} & \lesssim H^{r_{s}} \varepsilon_{h_{m}}^{*}\left(\lambda_{i}\right), s=0,1, \\
\left|\lambda_{i}-\lambda_{j, h_{m}}\right| & \lesssim \varepsilon_{h_{m}}\left(\lambda_{i}\right) \varepsilon_{h_{m}}^{*}\left(\lambda_{i}\right) .
\end{aligned}
$$

Then after one correction step, there exists two eigenpairs $\left(\widehat{u}_{j}, \widehat{w}_{j}\right),\left(\widehat{u}_{j}^{*}, \widehat{w}_{j}^{*}\right)$ such that the resultant approximations $\left(\lambda_{j, h_{m+1}}, u_{j, h_{m+1}}, w_{j, h_{m+1}}\right),\left(\lambda_{j, h_{m+1}}^{*}, u_{j, h_{m+1}}^{*}\right.$, $\left.w_{j, h_{m+1}}^{*}\right) \in \mathcal{C} \times \mathbf{H}_{h_{m+1}}$ has the following estimates

$$
\begin{aligned}
\left\|\left(u_{j, h_{m+1}}, w_{j, h_{m+1}}\right)-\left(\widehat{u}_{j}, \widehat{w}_{j}\right)\right\|_{\mathbf{H}} & \lesssim \varepsilon_{h_{m+1}}\left(\lambda_{i}\right), \\
\left\|\left(u_{j, h_{m+1}}, w_{j, h_{m+1}}\right)-\left(\widehat{u}_{j}, \widehat{w}_{j}\right)\right\|_{\mathbf{H}_{s}} & \lesssim H^{r_{s}} \varepsilon_{h_{m+1}}\left(\lambda_{i}\right), s=0,1, \\
\left\|\left(u_{j, h_{m+1}}^{*}, w_{j, h_{m+1}}^{*}\right)-\left(\widehat{u}_{j}^{*}, \widehat{w}_{j}^{*}\right)\right\|_{\mathbf{H}} & \lesssim \varepsilon_{h_{m+1}}^{*}\left(\lambda_{i}\right), \\
\left\|\left(u_{j, h_{m+1}}^{*}, w_{j, h_{m+1}}^{*}\right)-\left(\widehat{u}_{j}^{*}, \widehat{w}_{j}^{*}\right)\right\|_{\mathbf{H}_{s}} & \lesssim H^{r_{s}} \varepsilon_{h_{m+1}}^{*}\left(\lambda_{i}\right), s=0,1, \\
\left|\lambda_{i}-\lambda_{j, h_{m+1}}\right| & \lesssim \varepsilon_{h_{m+1}}\left(\lambda_{i}\right) \varepsilon_{h_{m+1}}^{*}\left(\lambda_{i}\right),
\end{aligned}
$$

where

$$
\varepsilon_{h_{m+1}}\left(\lambda_{i}\right):=\delta_{h_{m+1}}\left(\lambda_{i}\right)+H^{r_{0}} \varepsilon_{h_{m}}\left(\lambda_{i}\right)
$$

and

$$
\varepsilon_{h_{m+1}}^{*}\left(\lambda_{i}^{*}\right):=\delta_{h_{m+1}}^{*}\left(\lambda_{i}^{*}\right)+H^{r_{0}} \varepsilon_{h_{m}}^{*}\left(\lambda_{i}^{*}\right) .
$$

Proof. Since $\left\{\left(u_{j, h_{m+1}}, w_{j, h_{m+1}}\right)\right\}_{j=i}^{i+q-1}$ is a basis of $M_{h_{m}}\left(\lambda_{i}\right),\left\{\left(u_{j}, w_{j}\right)\right\}_{j=i}^{i+q-1}$ is a basis of $M\left(\lambda_{i}\right)$. For any $(v, z) \in M\left(\lambda_{i}\right)$ and $\|(v, z)\|_{\mathbf{H}}=1$ we denote

$$
(v, z)=\sum_{j=i}^{i+q-1} \gamma_{j}\left(u_{j}, w_{j}\right)
$$

For $\left(\widetilde{u}_{l, h_{m}}^{*}, \widetilde{w}_{l, h_{m}}^{*}\right) \in M_{h_{m}}\left(\lambda_{i}\right)$ in Assumption (A), thanks to (2.27) there exists $\left(\widetilde{u}_{l}^{*}, \widetilde{w}_{l}^{*}\right) \in M\left(\lambda_{i}\right)$ satisfying

$$
\left.\|\left(\widetilde{u}_{l}^{*}, \widetilde{w}_{l}^{*}\right)-\left(\widetilde{u}_{l, h_{m}}^{*}, \widetilde{w}_{l, h_{m}}^{*}\right)\right) \|_{\mathbf{H}} \lesssim \delta_{h_{m}}^{*}\left(\lambda_{i}^{*}\right) .
$$

Then

$$
A\left((v, z),\left(\widetilde{u}_{l}^{*}, \widetilde{w}_{l}^{*}\right)\right)=\sum_{j=i}^{i+q-1} \gamma_{j} A\left(\left(u_{j}, w_{j}\right),\left(\widetilde{u}_{l}^{*}, \widetilde{w}_{l}^{*}\right)\right) .
$$

Hence

$$
\left|\gamma_{l}\right|=\frac{1}{A\left(\left(u_{l}, w_{l}\right),\left(\widetilde{u}_{l}^{*}, \widetilde{w}_{l}^{*}\right)\right)}\left\{A\left((v, z),\left(\widetilde{u}_{l}^{*}, \widetilde{w}_{l}^{*}\right)\right)-\sum_{j=i, j \neq l}^{i+q-1} \gamma_{j} A\left(\left(u_{j}, w_{j}\right),\left(\widetilde{u}_{l}^{*}, \widetilde{w}_{l}^{*}\right)\right)\right\} .
$$

Due to Assumption (A) and $\left.\|\left(\widetilde{u}_{l}^{*}, \widetilde{w}_{l}^{*}\right)-\left(\widetilde{u}_{l, h_{m}}^{*}, \widetilde{w}_{l, h_{m}}^{*}\right)\right) \|_{\mathbf{H}} \lesssim \delta_{h_{m}}^{*}\left(\lambda_{i}^{*}\right)$, since

$$
\begin{aligned}
\left|A\left(\left(u_{j}, w_{j}\right),\left(\widetilde{u}_{l}^{*}, \widetilde{w}_{l}^{*}\right)\right)\right| \leq & \left|A\left(\left(u_{j}, w_{j}\right)-\left(u_{j, h_{m}}, w_{j, h_{m}}\right),\left(\widetilde{u}_{l}^{*}, \widetilde{w}_{l}^{*}\right)\right)\right| \\
& +\left|A\left(\left(u_{j, h_{m}}, w_{j, h_{m}}\right),\left(\widetilde{u}_{l}^{*}, \widetilde{w}_{l}^{*}\right)-\left(\widetilde{u}_{l, h_{m}}^{*}, \widetilde{w}_{l, h_{m}}^{*}\right)\right)\right| \\
& +\left|A\left(\left(u_{j, h_{m}}, w_{j, h_{m}}\right),\left(\widetilde{u}_{l, h_{m}}^{*}, \widetilde{w}_{l, h_{m}}^{*}\right)\right)\right| \\
\lesssim & \varepsilon_{h_{m}}\left(\lambda_{i}\right)+\delta_{h_{m}}^{*}\left(\lambda_{i}^{*}\right)+H^{r_{0}},
\end{aligned}
$$


we have

$$
\left|\gamma_{l}\right| \lesssim \frac{1}{\left|A\left(\left(u_{l}, w_{l}\right),\left(\widetilde{u}_{l}^{*}, \widetilde{w}_{l}^{*}\right)\right)\right|}\left\{1+\sum_{j=i, j \neq l}^{i+q-1}\left|\gamma_{j}\right|\left(\varepsilon_{h_{m}}\left(\lambda_{i}\right)+\varepsilon_{h_{m}}^{*}\left(\lambda_{i}^{*}\right)+H^{r_{0}}\right)\right\} .
$$

Since $\left|A\left(\left(u_{l, h_{m}}, w_{l, h_{m}}\right),\left(\widetilde{u}_{l, h_{m}}^{*}, \widetilde{w}_{l, h_{m}}^{*}\right)\right)\right|$ has a positive lower bound uniformly with respect to $h_{m}$, so does $\left|A\left(\left(u_{l}, w_{l}\right),\left(u_{l}^{*}, w_{l}^{*}\right)\right)\right|$. It is immediate that

$$
\sum_{l=i}^{i+q-1}\left|\gamma_{l}\right| \lesssim q+q \sum_{j=i}^{i+q-1}\left|\gamma_{j}\right|\left(\varepsilon_{h_{m}}\left(\lambda_{i}\right)+\varepsilon_{h_{m}}^{*}\left(\lambda_{i}^{*}\right)+H^{r_{0}}\right),
$$

from which it follows that

$$
\sum_{j=i}^{i+q-1}\left|\gamma_{j}\right| \lesssim 1
$$

We set $\alpha_{j}=\lambda_{j} / \lambda_{j, h_{m}}$. By virtue of the orthogonality of $P_{h_{m+1}}$ and boundedness of $B(\cdot, \cdot)$ we have

$$
\begin{aligned}
& \left\|\alpha_{j}\left(\widehat{u}_{j, h_{m+1}}, \widehat{w}_{j, h_{m+1}}\right)-P_{h_{m+1}}\left(u_{j}, w_{j}\right)\right\|_{\mathbf{H}}^{2} \\
& \quad \lesssim A\left(\alpha_{j}\left(\widehat{u}_{j, h_{m+1}}, \widehat{w}_{j, h_{m+1}}\right)-P_{h_{m+1}}\left(u_{j}, w_{j}\right), \alpha_{j}\left(\widehat{u}_{j, h_{m+1}}, \widehat{w}_{j, h_{m+1}}\right)-P_{h_{m+1}}\left(u_{j}, w_{j}\right)\right) \\
& \quad=A\left(\alpha_{j}\left(\widehat{u}_{j, h_{m+1}}, \widehat{w}_{j, h_{m+1}}\right)-\left(u_{j}, w_{j}\right), \alpha_{j}\left(\widehat{u}_{j, h_{m+1}}, \widehat{w}_{j, h_{m+1}}\right)-P_{h_{m+1}}\left(u_{j}, w_{j}\right)\right) \\
& \quad=B\left(\lambda_{j, h_{m}} \alpha_{j}\left(u_{j, h_{m}}, w_{j, h_{m}}\right)-\lambda_{j}\left(u_{j}, w_{j}\right), \alpha_{j}\left(\widehat{u}_{j, h_{m+1}}, \widehat{w}_{j, h_{m+1}}\right)-P_{h_{m+1}}\left(u_{j}, w_{j}\right)\right) \\
& \quad \lesssim\left\|\left(u_{j, h_{m}}, w_{j, h_{m}}\right)-\left(u_{j}, w_{j}\right)\right\|_{\mathbf{H}_{\mathbf{0}}}\left\|\alpha_{j}\left(\widehat{u}_{j, h_{m+1}}, \widehat{w}_{j, h_{m+1}}\right)-P_{h_{m+1}}\left(u_{j}, w_{j}\right)\right\|_{\mathbf{H}},
\end{aligned}
$$

which together with (3.3) yields

$$
\left\|\alpha_{j}\left(\widehat{u}_{j, h_{m+1}}, \widehat{w}_{j, h_{m+1}}\right)-P_{h_{m+1}}\left(u_{j}, w_{j}\right)\right\|_{\mathbf{H}} \lesssim H^{r_{0}} \varepsilon_{h_{m}}\left(\lambda_{j}\right) .
$$

Thanks to Lemma 2.2, applying spectral approximation theory yields

$$
\begin{aligned}
& \left\|\left(u_{j, h_{m+1}}, w_{j, h_{m+1}}\right)-\left(\widehat{u}_{j}, \widehat{w}_{j}\right)\right\|_{\mathbf{H}} \lesssim\left\|\left(\widehat{u}_{j}, \widehat{w}_{j}\right)-P_{h_{m+1}}\left(\widehat{u}_{j}, \widehat{w}_{j}\right)\right\|_{\mathbf{H}} \\
& \quad \sup _{\substack{(v, z) \in M\left(\lambda_{i}\right) \\
\|(v, z)\|_{\mathbf{H}}=1}} \inf _{\left.h_{m+1}, z_{h_{m+1}}\right) \in \mathbf{H}_{H, h_{m+1}}}\left\|(v, z)-\left(v_{h_{m+1}}, z_{h_{m+1}}\right)\right\|_{\mathbf{H}} \\
& \quad \lesssim \sup _{\gamma_{j}}\left\|\sum_{j=i}^{i+q-1} \gamma_{j}\left(\left(u_{j}, w_{j}\right)-\alpha_{j}\left(\widehat{u}_{j, h_{m+1}}, \widehat{w}_{j, h_{m+1}}\right)\right)\right\|_{\mathbf{H}} \\
& \quad \lesssim \sum_{j=i}^{i+q-1}\left\|\left(u_{j}, w_{j}\right)-P_{h_{m+1}}\left(u_{j}, w_{j}\right)+P_{h_{m+1}}\left(u_{j}, w_{j}\right)-\alpha_{j}\left(\widehat{u}_{j, h_{m+1}}, \widehat{w}_{j, h_{m+1}}\right)\right\|_{\mathbf{H}} \\
& \lesssim \delta_{h_{m+1}}\left(\lambda_{j}\right)+H^{r_{0}} \varepsilon_{h_{m}}\left(\lambda_{j}\right)=\varepsilon_{h_{m+1}}\left(\lambda_{j}\right),
\end{aligned}
$$

and for $s=0,1$,

$$
\begin{aligned}
& \left\|\left(u_{j, h_{m+1}}, w_{j, h_{m+1}}\right)-\left(\widehat{u}_{j}, \widehat{w}_{j}\right)\right\|_{\mathbf{H}_{\mathbf{s}}} \lesssim\left\|\left(\widehat{u}_{j}, \widehat{w}_{j}\right)-P_{h_{m+1}}\left(\widehat{u}_{j}, \widehat{w}_{j}\right)\right\|_{\mathbf{H}_{s}} \\
& \quad \lesssim H^{r_{s}}\left\|\left(\widehat{u}_{j}, \widehat{w}_{j}\right)-P_{h_{m+1}}\left(\widehat{u}_{j}, \widehat{w}_{j}\right)\right\|_{\mathbf{H}} \\
& \quad \lesssim H^{r_{s}} \varepsilon_{h_{m+1}}\left(\lambda_{j}\right),
\end{aligned}
$$

where we have used (2.20) in the second inequality above. The above argument implies (3.7)-(3.8) hold. Similarly we can also prove (3.9)-(3.10). Using the 
proof method to show (3.7), we can derive (3.11) from (2.29). 2 .

Now we are in a position to analyze the convergence of Multigrid Algorithm

Theorem 3.2. Suppose the conditions of Theorem 3.1 hold. Let the numerical eigenpair $\left(\lambda_{j, h_{N}}, u_{j, h_{N}}, w_{j, h_{N}}, u_{j, h_{N}}^{*}, w_{j, h_{N}}^{*}\right)(j=i, i+1, \cdots, i+q-1)$ be obtained by Algorithm 2. Then there exists an eigenpair $\left(\lambda_{i}, u_{i}, w_{i}, u_{i}^{*}, w_{i}^{*}\right)$ such that the following estimates hold

$$
\begin{aligned}
\left\|\left(u_{j, h_{N}}, w_{j, h_{N}}\right)-\left(u_{j}, w_{j}\right)\right\|_{\mathbf{H}} & \lesssim \delta_{h_{N}}(\lambda), \\
\left\|\left(u_{j, h_{N}}, w_{j, h_{N}}\right)-\left(u_{j}, w_{j}\right)\right\|_{\mathbf{H}_{s}} & \lesssim H^{r_{s}} \delta_{h_{N}}(\lambda), s=0,1, \\
\left\|\left(u_{j, h_{N}}^{*}, w_{j, h_{N}}^{*}\right)-\left(u_{j}^{*}, w_{j}^{*}\right)\right\|_{\mathbf{H}} & \lesssim \delta_{h_{N}}^{*}(\lambda), \\
\left\|\left(u_{j, h_{N}}^{*}, w_{j, h_{N}}^{*}\right)-\left(u_{j}^{*}, w_{j}^{*}\right)\right\|_{\mathbf{H}_{s}} & \lesssim H^{r_{s}} \delta_{h_{N}}(\lambda), s=0,1, \\
\left|\lambda_{i}-\lambda_{j, h_{N}}\right| & \lesssim \delta_{h_{N}}(\lambda) \delta_{h_{N}}^{*}(\lambda) .
\end{aligned}
$$

Proof. It's immediate from (2.27)-(2.29) that

$$
\begin{aligned}
\left\|\left(u_{j, h_{1}}, w_{j, h_{1}}\right)-\left(u_{j}, w_{j}\right)\right\|_{\mathbf{H}} & \lesssim \delta_{h_{1}}(\lambda), \\
\left\|\left(u_{j, h_{n}}, w_{j, h_{1}}\right)-\left(u_{j}, w_{j}\right)\right\|_{\mathbf{H}_{s}} & \lesssim H^{r_{s}} \delta_{h_{1}}(\lambda), s=0,1, \\
\left|\lambda-\lambda_{h_{1}}\right| & \lesssim \delta_{h_{1}}(\lambda) \delta_{h_{1}}^{*}(\lambda) .
\end{aligned}
$$

Let $\varepsilon_{h_{1}}\left(\lambda_{i}\right):=\delta_{h_{1}}\left(\lambda_{i}\right)$, due to $\delta_{h_{m}}\left(\lambda_{i}\right) \lesssim \beta \delta_{h_{m+1}}\left(\lambda_{i}\right)$, by recursion,

$$
\begin{aligned}
\varepsilon_{h_{N}}\left(\lambda_{i}\right) & =\delta_{h_{N}}\left(\lambda_{i}\right)+H^{r_{0}} \varepsilon_{h_{N-1}}\left(\lambda_{i}\right) \\
& \lesssim \delta_{h_{N}}\left(\lambda_{i}\right)+H^{2 r_{0}} \delta_{h_{N-1}}\left(\lambda_{i}\right)+H^{r_{0}} \varepsilon_{h_{N-2}}\left(\lambda_{i}\right) \\
& \lesssim \sum_{m=1}^{N} H^{(N-m) r_{0}} \delta_{h_{m}}\left(\lambda_{i}\right) \\
& \lesssim \sum_{m=1}^{N} H^{(N-m) r_{0}} \beta^{N-m} \delta_{h_{N}}\left(\lambda_{i}\right) \\
& \lesssim \frac{1}{1-H^{r_{0}} \beta} \delta_{h_{N}}\left(\lambda_{i}\right) \\
& \lesssim \delta_{h_{N}}\left(\lambda_{i}\right) .
\end{aligned}
$$

Likewise, denoting $\varepsilon_{h_{1}}^{*}\left(\lambda_{i}\right):=\delta_{h_{1}}^{*}\left(\lambda_{i}\right)$ we can prove $\varepsilon_{h_{N}}^{*}\left(\lambda_{i}\right) \lesssim \delta_{h_{N}}^{*}\left(\lambda_{i}\right)$. Using Theorem 3.1 we can obtain the desired results.

Remark 3.1. Similar to Section 5 of [12, we can estimate the computational work of Algorithm 2 and prove that solving the transmission eigenvalue problem needs almost the same work as solving the corresponding boundary value problem.

\section{Numerical experiment}

In this section, we will report some numerical experiments for solving the transmission eigenvalue problem (2.6) by multigrid method (Algorithm 2) to validate our theoretical results.

We use Matlab 2012a to solve (2.1)-(2.4) on a Lenovo G480 PC with 4G 
memory. Our program is compiled under the package of iFEM [34]. In Algorithms 2, we use internal command eigs in Matlab to solve matrix eigenvalue problem use command ' $\backslash$ ' in Matlab to solve the associated linear algebraic systems. In numerical examples, since the exact eigenvalues are unknown, we compute the relatively accurate ones to replace them.

In our numerical experiments, we construct the finite element space $\mathbf{H}_{H, h_{m+1}}$ as $U \times V=$ :

$$
\left(S^{H}+\operatorname{span}\left\{\widehat{u}_{j, h_{m+1}}, \widehat{u}_{j, h_{m+1}}^{*}\right\}_{j=i}^{i+q-1}\right) \times\left(S^{H}+\operatorname{span}\left\{\widehat{w}_{j, h_{m+1}}, \widehat{w}_{j, h_{m+1}}^{*}\right\}_{j=i}^{i+q-1}\right),
$$

so that the first equation in Step 2 of Algorithm 1 can be conveniently written as

$$
\begin{aligned}
& \left(\frac{1}{n-1} \Delta u_{j, h_{m+1}}, \Delta v\right)_{0}=\lambda_{j, h_{m+1}}\left\{\left(\nabla\left(\frac{1}{n-1} u_{j, h_{m+1}}\right), \nabla v\right)_{0}\right. \\
& \left.\quad+\left(\nabla u_{j, h_{m+1}}, \nabla\left(\frac{n}{n-1} v\right)\right)_{0}-\left(\frac{n}{n-1} w_{j, h_{m+1}}, v\right)_{0}\right\}, \quad \forall v \in U, \\
& \left(w_{j, h_{m+1}}, z\right)_{0}=\lambda_{j, h_{m+1}}\left(u_{j, h_{m+1}}, z\right)_{0}, \quad \forall z \in V .
\end{aligned}
$$

For reading conveniently, we use the following notations in our tables and figures:

$k_{j, h}=\sqrt{\lambda_{j, h}}$ : The $j t h$ eigenvalue obtained by Algorithm 2 on $\mathbf{H}_{H, h}$.

We consider the case when $D$ is the unit square $(0,1)^{2}$ or the L-shaped domain $(-1,1)^{2} \backslash[0,1) \times(-1,0]$ and the index of refraction $n=16,4,8+x_{1}-x_{2}$. We choose $S^{h}$ to be the Bogner-Fox-Schmit rectangle element (BFS element) space such that $S^{h} \subset H_{0}^{2}(D)$, and the numerical results are shown in Tables 1-2. Besides, we depict the error curves for the numerical eigenvalues (see Figures 1-3) based on the results in Tables 1-2.

According to the regularity theory, we know $u, w \in H^{4}(D)$ if $D$ is a square. When the ascent of $k$ is equal to 1: according to (3.16), the convergence order of the eigenvalue approximation $k_{j, h}$ is four. It is seen from Figures 1-3 that the convergence order of the numerical eigenvalues on the unit square domain is four, which coincides with the theoretical result.

Figures 1-3 indicates that on the L-shaped domain, the convergence order of the listed numerical eigenvalues is less than four. This fact suggests that their corresponding eigenfunctions on the non-convex domain do have singularities in different degrees.

In addition, Tables 1-2 show that the numerical eigenvalues obtained by Algorithm 2 using BFS elements give a good approximation; our multigrid method can achieve the same convergence order as the one in 12. It is worth noticing that our method can compute real and complex transmission eigenvalues efficiently (see Tables 1-2).

Submitted to Applied Mathematics and Computation on Oct 17, 2015

\section{References}

[1] C. Cakoni, D. Colton, P. Monk, J. Sun, The inverse electromagnetic scattering problem for anisotropic media, Inverse Problem, 26(2010), 074004. 
[2] D. Colton, R. Kress, Inverse Acoustic and Electromagnetic Scattering Theory, 2nd ed., Vol. 93 in Applied Mathematical Sciences, Springer, New York, 1998.

[3] F. Cakoni, M. Cayoren, D. Colton, Transmission eigenvalues and the nondestructive testing of dielectrics, Inverse Problems, 24, 065016 (2008)

[4] F. Cakoni, D. Gintides, H. Haddar, The existence of an infinite discrete set of transmission eigenvalues, SIAM J. Math. Anal., 42(2010), pp. 237-255

[5] A. Kirsch, On the existence of transmission eigenvalues, Inverse Probl. Imaging, 3(2), 155-172 (2009)

[6] L. PäIväRInta, J. Sylvester, Transmission eigenvalues, SIAM J. Math. Anal., 40(2)(2008), pp. 738-753

[7] B.P. Rynne, B.D. Sleeman, The interior transmission problem and inverse scattering from inhomogeneous media, SIAM J. Math. Anal., 22 (1991), pp. 1755-1762 (1992).

[8] F. Cakoni, H. Haddar, On the existence of transmission eigenvalues in an inhomogeneous medium, Appl. Anal. 88(4)(2009), pp. 475-493.

[9] D. Colton, L. Pärvärinta, J. Sylvester, The interior transmission problem, Inverse Problem Imaging, 1(2007), pp. 13-28.

[10] D. Colton, P. Monk, J. Sun, Analytical and computational methods for transmission eigenvalues, Inverse Problems, 26 (2010), 045011.

[11] J. Sun, Iterative methods for transmission eigenvalues, SIAM J. Numer. Anal., 49(2011), pp. 1860-1874.

[12] X. JI, J. Sun, H. XIE, A multigrid method for Helmholtz transmission eigenvalue problems, J. Sci. Comput., 60(2014), pp. 276-294.

[13] J. An, J. Shen, A spectral-element method for transmission eigenvalue problems, J. Sci. Comput., 57(2013), pp. 670-688.

[14] F. CAKoni, P. Monk, J. Sun, Error analysis for the finite element approximation of transmission eigenvalues, Computational Methods in Applied Mathematics, 14(2014), pp. 419-427.

[15] Y. YAng, J. Han, H. BI, A new weak formulation and finite element approximation for transmission eigenvalues, 2015, arXiv:1506.06486.

[16] J. Xu And A. Zhou, A two-grid discretization scheme for eigenvalue problems, Math. Comput.,70 (2001), pp. 17-25.

[17] Q. LIN, Some problems concerning approximate solutions of operator equations, Acta Math. Sinica, 22(1979), pp. 219-230 (in Chinese).

[18] J. Xu, A. ZHOU, Local and parallel finite element algorithms for eigenvalue problems, Acta Math. Appl. Sin. Engl. Ser., 18(2002), pp. 185-200.

[19] Y. Yang, H. BI, J. Han, Y. Yu, The Shifted-inverse Iteration Based on the Multigrid Discretization for Eigenvalue Problems, SIAM J. Numer. Anal., 2015, accepted for publication. 
[20] Y. YANG, J. HAn, The multilevel mixed finite element discretizations based on local defect-correction for the Stokes eigenvalue problem,Comput. Methods Appl. Mech. Engrg. 289 (2015), pp.249-266.

[21] X. Dai And A. Zhou, Three-scale finite element discretizations for quantum eigenvalue problems, SIAM J. Numer. Anal., 46(2008), pp. 295-324.

[22] Q. Lin, H. XIE, A multi-level correction scheme for eigenvalue problems, Math. Comp., 84(2015), pp. 71-88.

[23] H. XIE, A multigrid method for eigenvalue problem, J. Comp. Phys., 274(2014), pp. 550-561.

[24] N. Moës, J. Dolbow, T. Belytschko, A finite element method for crack growth without remeshing, Int. J. Numer. Meth. Eng., 46(1999), pp. 131-150.

[25] T. BelytschKo, T. Black, Elastic crack growth in finite elements with minimal remeshing, Int . J. Numer. Methods Eng., 45(1999), pp. 601-620.

[26] C. Duarte, J. Oden, An hp adaptive method using clouds, Comput. Methods Appl. Mech. Eng., 139(1996), pp. 237-262.

[27] J. Melenk, I. BABušKA, The partition of unity finite element method: basic theory and applications, Comput. Methods Appl. Mech. Eng., 39(1996), pp. 289-314.

[28] T. Belytschko, R. Gracie, G. Ventura, A review of extended/generalized finite element methods for material modeling, Modelling Simul. Mater. Sci. Eng., 17(2009), 043001.

[29] T. Fries, T. Belytschko, The extended/generalized finite element method: An overview of the method and its applications, Int. J. Numer. Meth. Eng., 84(2010), pp. 253-304.

[30] H. Blum, R. Rannacher, On the boundary value problem of the biharmonic operator on domains with angular corners, Math. Method Appl. Sci., 2(1980), pp. 556-581.

[31] I. Babuska, J. Osborn, Eigenvalue Problems, in: P .G. Ciarlet, J. L. Lions, (Ed.), Finite Element Methods (Part 1), Handbook of Numerical Analysis, vol.2, Elsevier Science Publishers, North-Holand, 1991, 640-787.

[32] F. Chatelin, Spectral Approximations of Linear Operators, Academic Press, New York, 1983.

[33] Z. Peng, H. BI, H. Li, Y. YAng, A multilevel correction method for convection-diffusion eigenvalue problems, Math. Prob. Eng., 2015(2015), 10 pages, http://dx.doi.org/10.1155/2015/904347.

[34] L. CHEn, An integrated finite element method package in MATLAB, Technical Report, University of California at Irvine, 2009. 
Table 1: The numerical eigenvalues obtained by Algorithm 2 on the unit square domain with $H=h_{1}=\frac{\sqrt{2}}{8}\left(n=16,8+x_{1}-x_{2}\right)$ and $H=h_{1}=\frac{\sqrt{2}}{16}(n=4)$.

\begin{tabular}{llllll}
\hline$n$ & $h$ & $k_{1, h}$ & $k_{2, h}, k_{3, h}$ & $k_{4, h}$ & \\
\hline 16 & $\frac{\sqrt{2}}{8}$ & 1.880051827 & 2.446255515 & 2.868193148 & \\
16 & $\frac{\sqrt{2}}{16}$ & 1.879621633 & 2.444371226 & 2.866560541 & \\
16 & $\frac{\sqrt{2}}{32}$ & 1.879593109 & 2.444244719 & 2.866446979 & \\
16 & $\frac{\sqrt{2}}{64}$ & 1.879591295 & 2.444236640 & 2.866439605 & \\
16 & $\frac{\sqrt{2}}{128}$ & 1.879591180 & 2.444236133 & 2.866439141 & \\
16 & $\frac{\sqrt{2}}{256}$ & 1.879591166 & 2.444236099 & 2.866439110 & \\
\hline \hline$n$ & $h$ & $k_{1, h}, k_{2, h}$ & & $k_{3, h}$ & $k_{5, h}$ \\
\hline 4 & $\frac{\sqrt{2}}{16}$ & $4.271570823 \pm 1.147502410 \mathrm{i}$ & 5.477120630 & 6.100857372 \\
4 & $\frac{\sqrt{2}}{32}$ & $4.271689022 \pm 1.147437637 \mathrm{i}$ & 5.476172735 & 6.100321070 \\
4 & $\frac{\sqrt{2}}{64}$ & $4.271696373 \pm 1.147433642 \mathrm{i}$ & 5.476112619 & 6.100286894 \\
4 & $\frac{\sqrt{2}}{128}$ & $4.271696834 \pm 1.147433395 \mathrm{i}$ & 5.476108843 & 6.100284742 \\
4 & $\frac{\sqrt{2}}{256}$ & $4.271696869 \pm 1.147433423 \mathrm{i}$ & 5.476108632 & 6.100284617 \\
\hline \hline$n$ & $h$ & $k_{1, h}$ & $k_{2, h}$ & $k_{5, h}, k_{6, h}$ & \\
\hline $8+x_{1}-x_{2}$ & $\frac{\sqrt{2}}{8}$ & 2.823445794 & 3.542452244 & $4.4971031374 \pm 0.8770188489 \mathrm{i}$ \\
$8+x_{1}-x_{2}$ & $\frac{\sqrt{2}}{16}$ & 2.822270903 & 3.538946571 & $4.4966538580 \pm 0.8718551076 \mathrm{i}$ \\
$8+x_{1}-x_{2}$ & $\frac{\sqrt{2}}{32}$ & 2.822194508 & 3.538712669 & $4.4965518150 \pm 0.8714987351 \mathrm{i}$ \\
$8+x_{1}-x_{2}$ & $\frac{\sqrt{2}}{64}$ & 2.822189665 & 3.538697695 & $4.4965525625 \pm 0.8714831407 \mathrm{i}$ \\
$8+x_{1}-x_{2}$ & $\frac{\sqrt{2}}{128}$ & 2.822189362 & 3.538696758 & $4.4965519527 \pm 0.8714818531 \mathrm{i}$ \\
$8+x_{1}-x_{2}$ & $\frac{\sqrt{2}}{256}$ & 2.822189348 & 3.538696701 & $4.4965519517 \pm 0.8714817941 \mathrm{i}$ \\
\hline
\end{tabular}

Table 2: The numerical eigenvalues obtained by Algorithm 2 on the L-shaped domain with $H=h_{1}=\frac{\sqrt{2}}{8}$.

\begin{tabular}{llllll}
\hline$n$ & $h$ & $k_{1, h}$ & $k_{2, h}$ & $k_{3, h}$ & $k_{4, h}$ \\
\hline 16 & $\frac{\sqrt{2}}{8}$ & 1.4850654 & 1.5705634 & 1.7078129 & 1.7834681 \\
16 & $\frac{\sqrt{2}}{16}$ & 1.4802424 & 1.5699011 & 1.7061982 & 1.7831490 \\
16 & $\frac{\sqrt{2}}{32}$ & 1.4780404 & 1.5697716 & 1.7055795 & 1.7831209 \\
16 & $\frac{\sqrt{2}}{64}$ & 1.4770116 & 1.5697385 & 1.7052950 & 1.7831171 \\
16 & $\frac{\sqrt{2}}{128}$ & 1.4765288 & 1.5697294 & 1.7051613 & 1.7831163 \\
\hline \hline$n$ & $h$ & $k_{1, h}, k_{2, h}$ & & $k_{3, h}$ & $k_{4, h}$ \\
\hline 4 & $\frac{\sqrt{2}}{8}$ & $3.1106061 \pm 1.1986040 \mathrm{i}$ & 3.5307679 & 3.6418388 \\
4 & $\frac{\sqrt{2}}{16}$ & $3.1061706 \pm 1.1925668 \mathrm{i}$ & 3.5239697 & 3.6391912 \\
4 & $\frac{\sqrt{2}}{32}$ & $3.1038283 \pm 1.1897655 \mathrm{i}$ & 3.5213679 & 3.6387478 \\
4 & $\frac{\sqrt{2}}{64}$ & $3.1027011 \pm 1.1884482 \mathrm{i}$ & 3.5202067 & 3.6386426 \\
4 & $\frac{\sqrt{2}}{128}$ & $3.1021669 \pm 1.1878286 \mathrm{i}$ & 3.5196692 & 3.6386141 \\
\hline \hline$n$ & $h$ & $k_{1, h}$ & $k_{2, h}$ & $k_{5, h}, k_{6, h}$ & \\
\hline $8+x_{1}-x_{2}$ & $\frac{\sqrt{2}}{8}$ & 2.3127184 & 2.3974892 & $2.9287347 \pm 0.5743458 \mathrm{i}$ \\
$8+x_{1}-x_{2}$ & $\frac{\sqrt{2}}{16}$ & 2.3069617 & 2.3960567 & $2.9272395 \pm 0.5686048 \mathrm{i}$ \\
$8+x_{1}-x_{2}$ & $\frac{\sqrt{2}}{32}$ & 2.3043809 & 2.3957864 & $2.9254909 \pm 0.5666799 \mathrm{i}$ \\
$8+x_{1}-x_{2}$ & $\frac{\sqrt{2}}{64}$ & 2.3031831 & 2.3957182 & $2.9247531 \pm 0.5656800 \mathrm{i}$ \\
$8+x_{1}-x_{2}$ & $\frac{\sqrt{2}}{128}$ & 2.3026210 & 2.3956994 & $2.9245398 \pm 0.5649109 \mathrm{i}$ \\
\hline
\end{tabular}



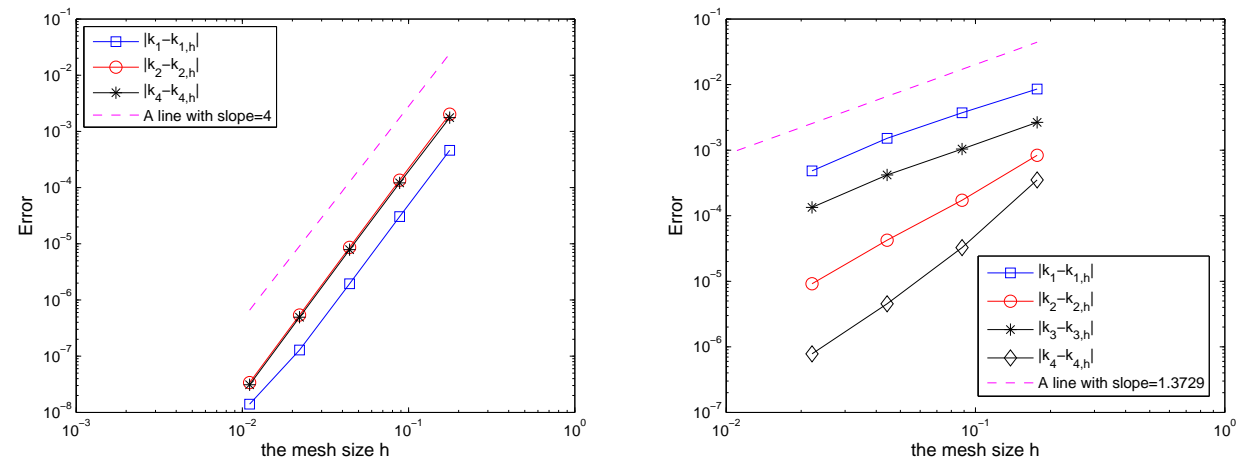

Figure 1: Error curves for numerical eigenvalues on the unit square(left) and on the L-shaped(right) with $n=16$.
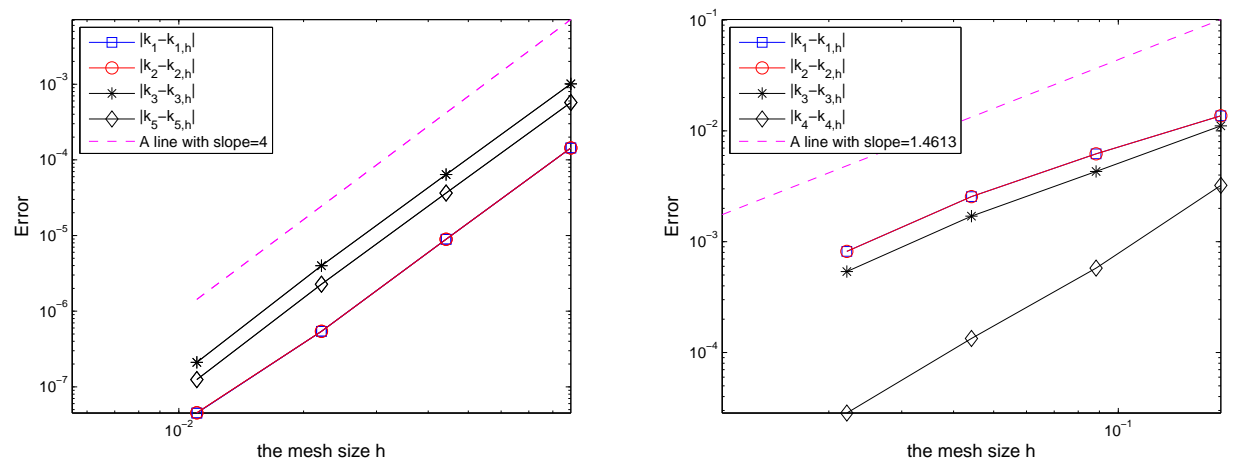

Figure 2: Error curves for numerical eigenvalues on the unit square(left) and on the L-shaped(right) with $n=4$.
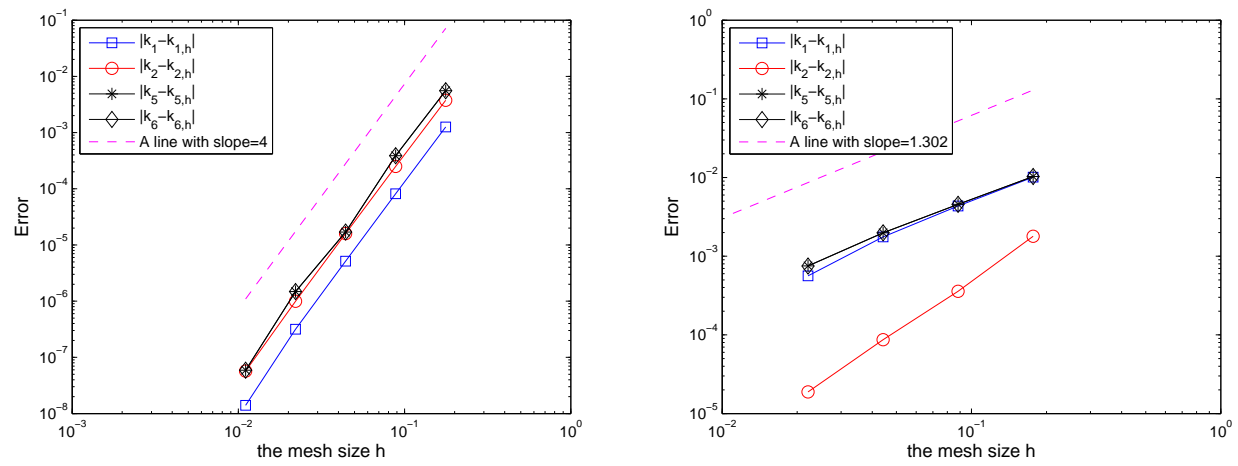

Figure 3: Error curves for numerical eigenvalues on the unit square(left) and on the L-shaped(right) with $n=8+x_{1}-x_{2}$. 